\title{
Evaluation of the influence of manufacturing errors on the quality of sealing joints installation
}

\author{
Aleksey Tarakhovskiy ${ }^{1, *}$, and Vadim Kramar $^{1}$ \\ ${ }^{1}$ Sevastopol State University, 299053, 33, Universitetskaya St.,Sevastopol, Russia
}

\begin{abstract}
In production process, the leading role belongs to installation as a final stage, where the main quality indicators, reliability and durability of the produced objects are formed. However, the analysis of machine building development trends in the recent years shows that even in such highly automated industries as automobile and tractor construction, in view of labor intensity, installation takes second place after machining. In the general structure of labor intensity of machine manufacture, it amounts to $35-60 \%$ of the total labor intensity and exceeds labor costs at all stages of production [1]. In machine building, about $25-30 \%$ of operations are mechanized, and no more than $5-6 \%$ of operations are automated [1]. Therefore, installation works are inefficient and expensive, resulting in the significant increase of the finished product cost and decrease of its quality.
\end{abstract}

The subunits in the form of "elastic ring element - internal groove" gained traction. In production, O-rings are installed into the internal groove of the cylindrical surface of the part by hand. During installation, the ring rotates around the axis of its cross-section, and during product operation, a rapid (20-100 operations) burst of the seal and loss of tightness takes place, which leads to failure of the entire structure, and the need for its unscheduled repair, while a high standard seal installation provides $5 \cdot 10^{3} \ldots 10^{9}$ cycles [2]. Analysis of the causes of O-rings failure shows that about $30 \%$ of defects occur due to the damage of O-rings during installation.

In the context of small-scale and medium-scale production, which is dominant in the domestic industry today, a high level of quality and efficiency of installation can be achieved through the development and introduction of new installation equipment.

The works by Gusev A.A., Golubev A.I., Zhitnikov Yu.Z., Ishchenko A.L., Kondakov L.A., Mikhailov A.N., Shabaykovich V.A., Shereshevskiy A.N., Yampolskiy L.S., Yakhimovich V.A. and some others are devoted to the research in the field of automation of elastic O-rings installation.

However, the issues of elastic ring deformation at the stages of installation, interworking and interference of the installed parts, the choice of rational positioning and installation equipment modularity were not fully investigated. The task of developing and researching the methods and means of installation acquires special significance if one

${ }^{*}$ Corresponding author : atarahovsky@gmail.com 
considers that about $35-40 \%$ of product failures in machine building industries are attributed to the units "elastic ring element - internal groove".

The quality of sealing joints (SJ) is regulated by a set of ISO standards, covering more than 30 indicators [3]. Most of them determine requirements for the cylinder and rod mating surfaces (tolerances for surface dimensions, shape deviations, surface roughness and undulation, etc.). However, it is possible to distinguish quality parameters, which depend on the conditions and features of installation operations. The main ones are:

No damage to the sealing joint surface.

1. No twisting (spiral twisting) of the sealing element.

2. No sealing joint deformations during installation, exceeding the permissible limit, when complete restoration of the ring shape becomes impossible.

3. No tilting of the sealing element in the groove.

4. No destruction of the sealing element.

A special feature of the sealing joint installation process is that the quality control of the joint after installation is almost impossible. For quality assessment, the benchmark test information is used, as well as data on the service of products containing such joints. To develop the requirements for mechanized and automated installation processes, a numerical evaluation of error probability when using one or another installation method is necessary.

The sealing joint installation conditions depend largely on the geometry of the installed parts. The influence of the base part characteristic can be reduced by performing all installation steps without contact of the elastic ring with the sealing surface. This justifies the necessity of carrying out the conjugation of the ring with the groove of the base part with a gap. In addition, when the ring is fed to the groove with a gap, the process of installation is simplified, which is especially important for the technology of flexible automated production. Installation of the ring in a free state, without twisting, also contributes to a significant increase in the quality, reliability and durability of the sealing device, and hence the quality of the machine as a whole.

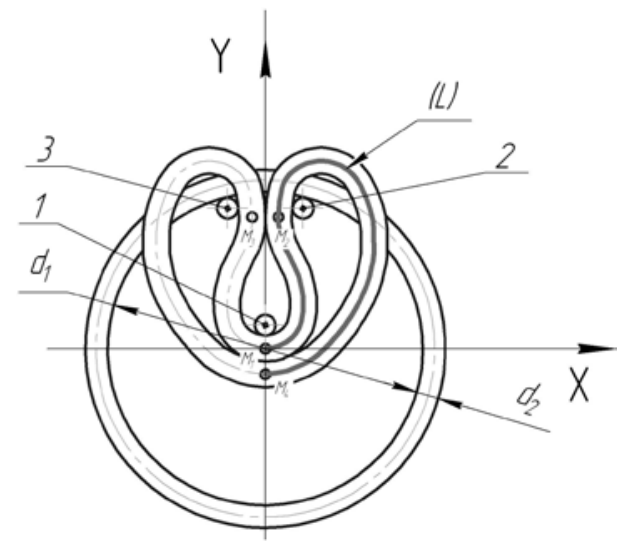

Fig. 1. Elastic line of the deformed ring with the minimum radius.

In this regard, our priority is the choice of the ring deformation method for effective reduction of its overall dimensions, which would allow us to inscribe the ring into a cylindrical boundary (CB) with a gap. This task is solved when the ring is bent with a protrusion facing inward. The corresponding deformation is shown in Fig. 1. Here, external fixed stop 1 ensures the inward bending of the ring, so corresponding point M1 of the elastic line approaches point M4 of the opposite section; internal fixed stops 2 and 3 are brought to one another to the full and the symmetrical sections of the ring touch at corresponding points M2 and M3. After deformation, the ring can be fed to the groove of 
the base part by the deforming body without contact with the sealing surface. The possibility of destruction or twisting of the ring is excluded, since the ring is fed to the groove with a gap. The rigidity of the ring is also increased, as the ring is fixed with the stops of the deforming body. In such conditions, the complex of the actuator and the elastic ring deformed by it can be considered as a rigid connecting part.

In accordance with installation conditions, the deformation of the elastic ring with a protrusion facing inward makes sense if, after the deformation, the ring can be inscribed into $\mathrm{CB}$ with the required gap. Since the radius of the circumscribed circle characterizes the dimensions of the deformed ring, then in order to mate with the gap, the following condition must be fulfilled:

$$
\widetilde{R}_{y \text { on }}-\left(\widetilde{R}_{\text {on }}^{\min }+0,5 \cdot d_{2}\right)-\Delta_{\Sigma} \geq C, m m
$$

where $R_{y}$ on - radius of the CB cross-section; $d_{2}$ - diameter of the ring cross-section; $\Delta_{\Sigma}$ - total relative orientation error; $C$ - minimum guaranteed gap.

To assess the possibility of mating with the gap, it is, first of all, necessary to determine radius $\widetilde{R}_{y \text { on }}$ of the cylindrical boundary cross-section, which can be obtained from the tables of DSTU GOST 24191: 2008.

Radius $\widetilde{R}_{\text {On }}^{\min }$ of the circumscribed circle, which depends on the geometric parameter of ring $\cdot v$ is calculated using the following formula:

$$
\widetilde{R}_{\text {On }}^{\min }=R \cdot(0,483 \cdot v+0,663),
$$

where $v$ - diameter $d_{2}$ of the ring cross-section, referenced to main radius $R$ $\left(R=\frac{d_{1}+d_{2}}{2}\right)$.

The methods of SJ installation with a gap created beforehand in mating are different from the known installation methods, as the process of ring mating occurs without contact with the base part surfaces (i.e., without contact with $\mathrm{CB}$ ). It is for this reason that schemes of mechanisms with relative mobility of the base or attached parts can not be applied. Methods and means of directional alignment and installation of parts can be applied, however, for SJ installation they will be characterized by considerable complexity and cumbersomeness.

The location schemes of SJ parts, like the entire installation process, differ in a number of features. First of all, the mechanisms should be characterized by rigid location and rigid relative installation of the assembled parts. When installing the sealing ring into the groove of the hole, the enclosing part is a part with a groove, and the enclosed part is an elastic sealing ring. More often, the enclosing part is based along the exterior auxiliary surface. For the enclosed mating surface of the connecting shaft, we take a conditional cylindrical surface with the radius of the cross section equal to radius $\widetilde{R}_{o n}^{\min }$ of the circle circumscribed around the ring deformed at the stops.

The total installation error (relative orientation error) is selected and calculated in each specific case. These matters are considered in numerous published papers [4].

If the mounting surface of the base part is cylindrical and the part is fixed with a movable prismatic clamp to a fixed plane, the relative orientation error $\Delta_{\Sigma}$ will be equal to: 


$$
\Delta_{\Sigma}=\varepsilon_{n p}+\varepsilon_{\sigma}+\varepsilon_{O}+\frac{\delta_{O}}{2}+\frac{\delta_{\text {on }}}{2}+\varepsilon_{v}, M M
$$

where $\varepsilon_{n p}$ - position error; $\varepsilon_{\sigma}-$ location error of the part, $\varepsilon_{\sigma}=\delta_{H} / 2 ; \delta_{H}$ - outer diameter tolerance of the base part; $\varepsilon_{o}$ - scale of the hole center displacement relative to the center of the external diameter of the base part; $\delta_{o}$ - tolerance for the sealing hole diameter (for the CB cross-section diameter); $\delta_{\text {on }}$ - deviation of the circumscribed circle radius; $\varepsilon_{v}$ - value of the circumscribed circle center displacement.

The distance between the external and internal stops, the ordinate of the circumscribed circle center, and the distance between the external stop and the circumscribed circle center depend only on the geometric parameter $\cdot v$, i.e. on the sizes $\left(d_{1}\right.$ and $\left.d_{2}\right)$ of the O-ring. The scattering of errors of these values, the variation of which depends on a large number of factors that do not have a predominant value, obeys the Gauss' law [5].

Let us estimate the mean square deviations of the calculated parameters. Let $Y=\varphi\left(X_{1}, X_{2}, \ldots, X_{n}\right)$ be function $n$ of random uncorrelated arguments $X_{1} \ldots X_{n}$. Assuming a small effect of the argument deviation on the function deviation, the indicated dependence can be linearized. The average quadratic deviation $\delta_{y}$ for a linear function according to [6] is expressed by the following formula:

$$
\delta_{y}^{2}=\sum_{i=1}^{n}\left(\frac{\partial \bar{\varphi}}{\partial X_{i}}\right)^{2} \delta X_{i}^{2}
$$

where $\frac{\partial \bar{\varphi}}{\partial X_{i}}$ are derivatives calculated for the value of the arguments equal to their mathematical expectations. Let us use (4) to determine the average quadratic deviation of the obtained dependences:

$$
\widetilde{R}_{\text {on }}^{\min }=\frac{d_{1}+d_{2}}{2} \cdot R_{\text {on }}^{\min }(v) .
$$

Thus, from (4) we will get:

$$
\delta_{\text {on }}^{2}=\left(\frac{\partial \widetilde{R}_{\text {on }}^{\min }}{\partial d_{1}}\right)^{2} \cdot \delta_{1}^{2}+\left(\frac{\partial \widetilde{R}_{o n}^{\min }}{\partial d_{2}}\right)^{2} \cdot \delta_{2}^{2},
$$

where $\delta_{o n}, \delta_{1}, \delta_{2}$ are average quadratic deviations, respectively, of the circumcised circle radius, internal diameter $d_{1}$ of the ring and cross-section diameter $d_{2}$ of the ring.

Let us remind you that $v=\frac{2 \cdot d_{2}}{d_{1}+d_{2}}$. 
We express $\frac{\partial \widetilde{R}_{o n}^{\min }}{\partial d_{1}}$ and $\frac{\partial \widetilde{R}_{o n}^{\min }}{\partial d_{2}}$, and substitute into equation (6).

We obtain taking into account (5):

$$
\begin{aligned}
& \frac{\partial \widetilde{R}_{O n}^{\min }}{\partial d_{1}}=\frac{1}{2} \cdot R_{\text {on }}^{\min }(v)+\frac{d_{1}+d_{2}}{2} \cdot \frac{\partial R_{O n}^{\min }}{\partial v} \cdot \frac{\partial v}{\partial d_{1}}= \\
& =\frac{1}{2} \cdot R_{o n}^{\min }(v)-\frac{d_{1}+d_{2}}{2} \cdot \frac{\partial R_{o n}^{\min }}{\partial v} \cdot \frac{2 \cdot d_{2}}{\left(d_{1}+d_{2}\right)^{2}}=\frac{1}{2}\left[R_{o n}^{\min }(v)-\frac{\partial R_{o n}^{\min }}{\partial v} \cdot v\right] \\
& \frac{\partial \widetilde{R}_{O n}^{\min }}{\partial d_{2}}=\frac{1}{2} \cdot R_{\text {on }}^{\min }(v)+\frac{d_{1}+d_{2}}{2} \cdot \frac{\partial R_{O n}^{\min }}{\partial v} \cdot \frac{\partial v}{\partial d_{1}}= \\
& =\frac{1}{2} \cdot R_{o n}^{\min }(v)-\frac{d_{1}+d_{2}}{2} \cdot \frac{\partial R_{o n}^{\min }}{\partial v} \cdot \frac{d_{1}+d_{2}-d_{2}}{\left(d_{1}+d_{2}\right)^{2}}=\frac{1}{2}\left[R_{o n}^{\min }(v)-\frac{\partial R_{o n}^{\min }}{\partial v} \cdot\left(1-\frac{v}{2}\right)\right] \\
& \delta_{\text {on }}^{2}=\frac{1}{4} \cdot\left[(0,48 v+0,66-0,48 v)^{2} \delta_{2}^{2}+(0,48 v+0,66+0,48-0,24 v)^{2} \delta_{2}^{2}\right]= \\
& =0,11 \delta_{1}^{2}+(0,12 \cdot v+0,57)^{2} \delta_{2}^{2}
\end{aligned}
$$

Similarly, we get:

$$
\begin{aligned}
& \delta_{v}^{2}=\frac{1}{4}\left[(0,66-0,52 v+0,52 v)^{2} \delta_{2}^{2}+(0,66-0,52 v-0,52+0,26 v)^{2} \delta_{2}^{2}\right]= \\
& =0,11 \delta_{1}^{2}+(0,14-0,26 \cdot v)^{2} \delta_{2}^{2} \\
& \delta_{H^{*}}^{2}=\frac{1}{4}\left[(1,15-0,58 v+0,58 v)^{2} \delta_{1}^{2}+(1,15-0,58 v-0,58+0,29 v)^{2} \delta_{2}^{2}\right]= \\
& =0,33 \delta_{1}^{2}+(0,57-0,29 \cdot v)^{2} \delta_{2}^{2}
\end{aligned}
$$

When the random variable is distributed according to the Gauss' law, the value of $6 \delta$ [5] is taken to be practically the limiting scattering field. Then we will have $\delta_{1}=\frac{1}{6} \cdot \delta \cdot d_{1}$, $\delta_{2}=\frac{1}{6} \cdot \delta \cdot d_{2}$, and

$$
\begin{gathered}
\delta_{\text {on }}=6 \delta_{\text {on }}=6 \sqrt{0,11 \delta_{1}^{2}+(0,12 \cdot v+0,57)^{2} \delta_{2}^{2}} ; \\
\varepsilon_{v}=6 \delta_{v}=6 \sqrt{0,11 \delta_{1}^{2}+(0,14-0,26 \cdot v)^{2} \delta_{2}^{2}} .
\end{gathered}
$$


The dependencies obtained allow to carry out calculations of the installation modes and the installation device elements.

\section{References}

1. Mechanical engineering. Encyclopedia / Editorial Board: K.V. Frolov (chair) and others. M.: Mechanical Engineering. Technology of assembly in engineering. V. III-5 / [A.A. Gusev, V.V. Pavlov, A.G. Andreev, etc.]; under the gen. ed. of Yu.M. Solomentsev. (2001). $640 \mathrm{p}$.

2. V.V. Burenin, V.P. Ivanin Durability evaluation of rubber sealing rings and cuffs for rotating shafts of machines and mechanisms Chem. and oil and gas. engineering. No. 11. - pp. 3233. (2003)

3. Quality management systems. Basic Terms and Glossary of Terms (ISO 9000:2005, IDT): DSTU ISO 9000:2007

4. L.A. Vakhrin, V.K. Myasnikov, V.T. Sinitsyn Adaptive control for automatic joint assembly Installation in machine building, instrument making, No. 1. P. 17-26,(2001)

5. V.I. Kostyuk, L.S. Yampolskiy. Flexible robotic systems. General approach, K.: Higher education, 1988. $72 \mathrm{P}$.

6. Automation of production processes in mechanical engineering and instrument making: Ukr. interdep. sci.-tech. collection / Ministry of Education and Science of Ukraine, National University "Lviv Polytechnic". Lviv: Ed. office of National University “Lviv Polytechnic”, 1965, Ed. 40 / Ed. by Stotsko. 2006. 312 p. 